\title{
FRET characterisation for cross-bridge dynamics in single-skinned rigor muscle fibres
}

\author{
Valentina Caorsi $\cdot$ Dmtry S. Ushakov \\ Timothy G. West $\cdot$ Niovi Setta-Kaffetzi • \\ Michael A. Ferenczi
}

Received: 28 June 2010/Revised: 9 August 2010/ Accepted: 16 August 2010/Published online: 2 September 2010

(C) The Author(s) 2010. This article is published with open access at Springerlink.com

\begin{abstract}
In this work we demonstrate for the first time the use of Förster resonance energy transfer (FRET) as an assay to monitor the dynamics of cross-bridge conformational changes directly in single muscle fibres. The advantage of FRET imaging is its ability to measure distances in the nanometre range, relevant for structural changes in actomyosin cross-bridges. To reach this goal we have used several FRET couples to investigate different locations in the actomyosin complex. We exchanged the native essential light chain of myosin with a recombinant essential light chain labelled with various thiol-reactive chromophores. The second fluorophore of the FRET couple was introduced by three approaches: labelling actin, labelling SH1 cysteine and binding an adenosine triphosphate (ATP) analogue. We characterise FRET in rigor cross-bridges: in this condition muscle fibres are well described by a single FRET population model which allows us to evaluate the true FRET efficiency for a single couple and the consequent donor-acceptor distance. The results obtained are in good agreement with the distances
\end{abstract}

Electronic supplementary material The online version of this article (doi:10.1007/s00249-010-0624-9) contains supplementary material, which is available to authorised users.

V. Caorsi ( $\square)$

Newton International Fellow, Royal Society, London, UK

e-mail: v.caorsi@imperial.ac.uk

V. Caorsi · D. S. Ushakov - T. G. West · N. Setta-Kaffetzi ·

M. A. Ferenczi

Molecular Medicine Section, NHLI, Imperial College London,

London, UK

Present Address:

T. G. West

Structure and Motion Laboratory, Royal Veterinary College,

University of London, London, UK expected from crystallographic data. The FRET characterisation presented herein is essential before moving onto dynamic measurements, as the FRET efficiency differences to be detected in an active muscle fibre are on the order of $10-15 \%$ of the FRET efficiencies evaluated here. This means that, to obtain reliable results to monitor the dynamics of cross-bridge conformational changes, we had to fully characterise the system in a steady-state condition, demonstrating firstly the possibility to detect FRET and secondly the viability of the present approach to distinguish small FRET variations.

Keywords FRET - Acceptor photobleaching · Spectral analysis - FLIM - Rigor muscle fibre . Actomyosin interactions

\section{Introduction}

Myosin is a motor protein which converts chemical energy into mechanical work and heat by cyclically interacting with actin filaments in an ATP-dependent manner. Several structural studies converged on the "lever arm" model of muscle contraction, where mechanical force is produced by conformational changes in the catalytic domain of the myosin head, followed by rotation of the regulatory domain which acts as a lever arm, capable of amplifying those conformational changes to produce force and movement (Geeves and Holmes 2005). In addition, the actin-myosin interface is likely to play an active role in force generation, as the attachment mode of myosin to actin changes during the cross-bridge cycle (Ferenczi et al. 2005).

However, the mechanism of the energy transduction process remains unclear; the conformational changes in the myosin molecule and at the actomyosin interface, the 
relationship between these structural changes, the ATPase cycle and the strain on the cross-bridges remain to be elucidated.

Various approaches are traditionally followed to study these mechanisms, namely electron microscopy, X-ray crystallography, low-angle X-ray diffraction and spectroscopic studies. Electron microscopy has played a key role in the investigation of muscle contraction, allowing visualisation of the structure and interaction of thick and thin filaments in sections of fixed, stained and embedded tissue (Huxley 1969). In addition, cryo-electron microscopy allows examination of the structure of frozen, hydrated sections of muscle fibre (Liu et al. 2004; McDowall et al. 1984). X-ray crystallographic measurements in protein crystals provide the ultimate description of protein complexes at the atomic scale, but require crystals from purified proteins extracted from muscle or from protein fragments expressed in recombinant systems which give a snapshot of a protein state frozen in time (Rayment et al. 1993a, b; Dominguez et al. 1998). The main limitation of electron microscopy and X-ray crystallography arises from the need to examine non-functional samples. Low-angle $\mathrm{X}$-ray diffraction allows study of living muscle fibres during contraction with high spatial and time resolution, but quantitative analysis of the diffraction pattern requires appropriate mathematical models, and often a unique interpretation is not possible (Dobbie et al. 1998; Koubassova et al. 2008). Spectroscopic studies of proteins in solution provide dynamic information about structural changes, but the behaviour of these isolated proteins may be different from in their natural, highly ordered context (Thomas 1987; Nyitrai et al. 2000a, b; Sun et al. 2008).

To investigate these structural changes directly in muscle fibres, we propose to exploit the power of Förster resonance energy transfer (FRET) to measure distances on the nanometre scale, coupled with the capability of confocal optical microscopy to observe the molecular components of muscle fibres in three dimensions without perturbing the system. In solution studies, FRET provides useful information on the global behaviour of active motor proteins (Moss and Trentham 1983; Botts et al. 1984; Ishiwata et al. 1997; Kast et al. 2010), for example to probe the lever arm hypothesis. In muscle fibres, FRET has the additional advantage that it can be coupled with force measurements, thus providing a novel time-resolved approach to correlate structural changes by means of fluorescence variations with force production.

Here, we investigate the rigor state in single permeabilised skeletal muscle fibres, positioning donor-acceptor pairs at strategic locations in the protein complex to detect myosin head conformational changes. The choice of the following three particular interactions was determined by our interest in the proximal part of the lever arm, which forms an interface with the catalytic domain. The three interaction we have investigated are: (1) between Cys707 on the SH1 helix and Cys180 on the essential light chain (ELC), and interaction of the same ELC locus (2) with the nucleotide binding pocket and (3) with actin (Fig. 1). FRET measurements were performed by means of both intensitybased and time-resolved methods. The acceptor photobleaching method (Kenworthy 2001) as well as spectral analysis (Zimmermann 2005) were used. A single FRET population model was applied to describe the muscle system in a rigor state, to evaluate the true FRET efficiency for a single couple and the consequent donor-acceptor distance. Indeed, the measured FRET efficiency estimated from intensity-based measurements depends on the relative donor-acceptor ratio. Lifetime measurements were used as a control on the intensity-based FRET results, as lifetime measurements are independent of fluorophore concentration

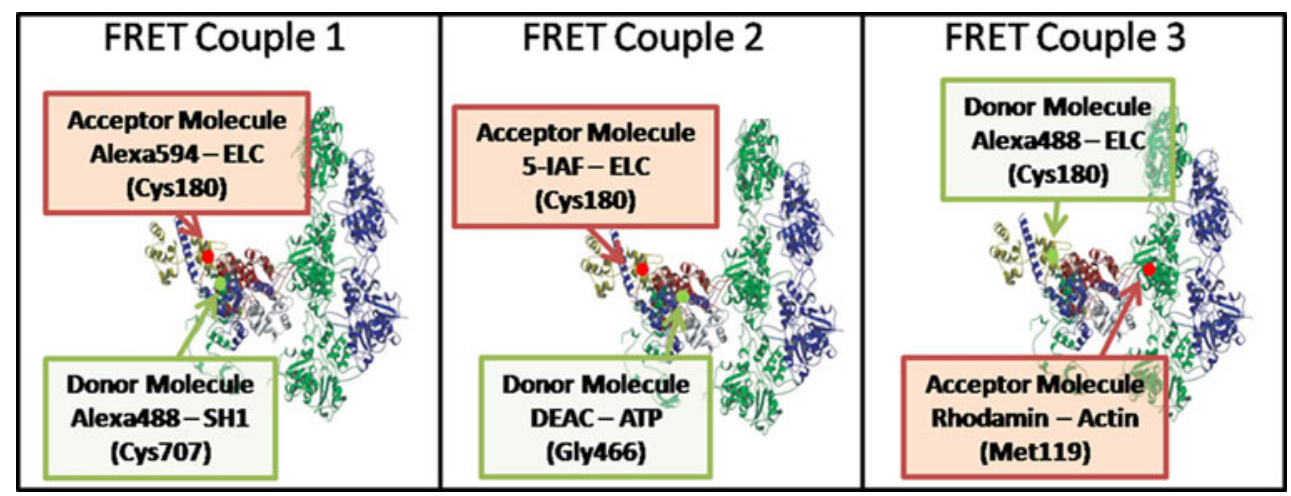

Fig. 1 Scheme showing the positions labelled to study FRET interactions between the ELC and SH1 helix (FRET couple 1), between the ELC and the nucleotide binding pocket (FRET couple 2) and between the ELC and actin (FRET couple 3). In the diagram (Rayment et al. 1993b) five actin molecules are shown in blue and green; the myosin head S1 presents a further colour-coded segmentation: ELC (yellow), 25-k N-terminal fragment (green), upper 50-k fragment (red), lower 50-k fragment (grey) and 20-k C-terminal fragment (blue) 
(Becker et al. 2006). The FRET efficiencies evaluated in these experiments allowed us to build up a distances map which is in agreement with the crystallographic data, thus demonstrating the viability of the present approach as a method for revealing structural features at the cross-bridge level. The capability to distinguish small FRET variations (the theoretical D-A distance is nearly $4 \mathrm{~nm}$ for ELC-SH1 interaction and $5 \mathrm{~nm}$ for ELC-DEAC-ATP) shows the good sensitivity of the present approach, which will be essential to monitor cross-bridge conformational changes.

\section{FRET model for rigor fibres}

FRET has been extensively used in many research areas as a "nanometric ruler" to investigate protein-protein interactions, to measure distances between two sites on a macromolecule and to follow conformational changes, protein folding and unfolding, etc. (Stryer 1978; Clegg 2002). Although FRET represents a powerful tool, FRET interpretation is often complicated by uncertainty about donor-acceptor stoichiometry. Different donor and acceptor concentrations affect FRET efficiency, as there may be unpaired donor and acceptor molecules which contribute to the detected intensities; there may be multiple interactions where one single donor molecule interacts with several acceptor molecules, or several donor molecules interact with fewer acceptor molecules. Quantitative analysis is required to distinguish between these various interactions, and to derive the time-averaged donor-acceptor distance and the fraction of interacting molecules from FRET measurements (Neher and Neher 2004).

Muscle fibres are a well-organised system with specialised molecular motors arranged in precise geometric arrays to maximise performance. This precise organisation simplifies the FRET model. In particular, we can neglect multiple interactions, as cross-bridge formation shows a pitch of $42.9 \mathrm{~nm}$ and an axial repeat of $14.3 \mathrm{~nm}$ (Squire 1997), distances which are too large for FRET to occur. ${ }^{1}$ Furthermore, we need to consider whether, in mammalian muscle fibre, all the donors that interact with an acceptor are interacting in the very same way, namely at the very same distance and the very same orientation. In many cases this would not be a justifiable assumption; however, in the rigor state studied here we can assume that all the myosin globular heads are tightly attached to actin in a specific conformation, thus forming a single population of FRET

\footnotetext{
${ }^{1}$ Since both myosin heads of a myosin molecule may be labelled by the fluorophores, the possibility of inter-head FRET and therefore of multiple interactions needs to be considered, and a different FRET theory should be applied accordingly. However, the results obtained are in agreement with the crystallographic expectations, suggesting that each head behaves independently and that FRET distances across two heads are probably too large for FRET detection.
}

pairs (Thomas and Cook 1980). Still, the contribution of unpaired donor and acceptor molecules to the fluorescent signals needs to be considered, as not all myosins may contain a D-A pair.

The average measured FRET efficiency $(\langle E\rangle)$ is linked to the true FRET efficiency of a single interacting couple $\left(E_{\text {single }}\right)$ by Eq. 1 if evaluated by detecting donor fluorescence variations, or by Eq. 2 if following acceptor fluorescence variations (Wouters et al. 2001; Gordon et al. 1998):

$$
\begin{aligned}
& \langle E\rangle_{\mathrm{D}}=\frac{m_{\mathrm{D}}}{n_{\mathrm{D}}} E_{\text {single }} \\
& \langle E\rangle_{\mathrm{A}}=\frac{\varepsilon_{\mathrm{D}}}{\varepsilon_{\mathrm{A}}} \frac{m_{\mathrm{A}}}{n_{\mathrm{A}}} E_{\text {single }},
\end{aligned}
$$

where $n_{\mathrm{D}}$ and $n_{\mathrm{A}}$ are the number of donor and acceptor molecules in the system, $m_{\mathrm{D}}=m_{\mathrm{A}}$ is the number of interacting donors/acceptors, and $\varepsilon_{\mathrm{D}}$ and $\varepsilon_{\mathrm{A}}$ are the donor and acceptor extinction coefficients, respectively. It is worth underlining the major assumptions for which these equations are valid: all the interacting molecules are interacting in the very same way (single FRET population), and multiple interactions are neglected. Equation 1 states that, if all the donors are interacting, $m_{\mathrm{D}}=n_{\mathrm{D}}$, the measured FRET efficiency is equal to the FRET efficiency of the single FRET couple, and the whole system is behaving as a single couple. However, if a fraction of the donors are not interacting (which is the most likely case), the measured FRET efficiency underestimates the FRET efficiency of interacting pairs. Similar considerations relate to Eq. 2: a further factor $\left(\varepsilon_{\mathrm{D}} / \varepsilon_{\mathrm{A}}\right)$ is needed here to take into account the different excitation wavelengths used to evaluate the measured FRET efficiency (see Appendix for further details).

These relations are clearly important for evaluation of the true donor-acceptor distance, which is given by:

$r=R_{0} \sqrt[6]{\frac{1-E_{\text {single }}}{E_{\text {single }}} .}$

The Förster radius $R_{0}$ depends on the orientation factor (see "Materials and methods": FRET couple characterisation, Eq. 4). In the present study, both the donor and acceptor are assumed to rotate freely in a time that is short compared with the excited-state lifetime of the donor.

\section{Materials and methods}

Reagents and solutions

Table 1 lists the solutions used in the experiments. AlexaFluor488 maleimide, AlexaFluor594 maleimide, rhodamine phalloidin and $5^{\prime}$-iodoacetamidofluorescein 
Table 1 Solutions

\begin{tabular}{|c|c|c|c|c|}
\hline & $\begin{array}{l}\text { Relaxing } \\
\text { solution }\end{array}$ & $\begin{array}{l}\text { Pre-rigor } \\
\text { solution }\end{array}$ & $\begin{array}{l}\text { Ca-rigor } \\
\text { solution }\end{array}$ & $\begin{array}{l}\text { Exchange } \\
\text { solution }\end{array}$ \\
\hline ATP & 7 & & & 5 \\
\hline EGTA & 5 & 30 & 0.25 & 5 \\
\hline $\mathrm{Ca}^{2+}$ EGTA & & & 29.75 & \\
\hline EDTA & & & & 5 \\
\hline $\mathrm{Mg}$ acetate & 8 & & & \\
\hline Imidazole & 6 & & & 10 \\
\hline K-propionate & 70 & 20.64 & 23.28 & 150 \\
\hline TES & & 60 & 60 & \\
\hline $\mathrm{MgCl}_{2}$ & & 4.5 & 2.02 & \\
\hline Glutathione & & 20 & 20 & \\
\hline $\begin{array}{l}\text { 2,3-Butanedione } \\
\text { 2-monoxime (BDM) }\end{array}$ & & 10 & & \\
\hline $\mathrm{KH}_{2} \mathrm{PO}_{4}$ & & & & 10 \\
\hline DTT & & & & 5 \\
\hline Trifluoroperazine (TFP) & & & & 0.5 \\
\hline $\mathrm{pH}$ & 7.1 & 7.1 & 7.1 & 6.5 \\
\hline
\end{tabular}

All values (except $\mathrm{pH}$ ) in $\mathrm{mM}$

EGTA, ethylene glycol tetraacetic acid; EDTA, ethylenediamine tetraacetic acid; TES, N-tris[hydroxymethyl] ethyl-2-aminoethanesulfonic acid; DTT, Dithiothreitol

(5-IAF) were obtained from Invitrogen, UK. $3^{\prime}-O-\{N-[3-$ (7-diethylaminocoumarin-3-carboxamido)propyl]carbamoyl \}ATP (DEAC-pda-ATP) was obtained from M. Webb and G. Reid (National Institute for Medical Research, London) (Webb et al. 2004; Webb and Corrie 2001). All other chemicals and reagents were from Sigma-Aldrich, UK.

Protein labelling

The mutated ELC-180 (Borejdo et al. 2001) was labelled with different thiol-reactive dyes, namely AlexaFluor 488 maleimide, AlexaFluor594 maleimide and 5'-iodoacetamidofluorescein (5-IAF) (Invitrogen, UK). In all cases, the protein was mixed with the fluorophore at 1:3 $\mathrm{M}$ ratio in a solution containing $10 \mathrm{mM} \mathrm{K}$-phosphate, $100 \mathrm{mM} \mathrm{KCl}$, $\mathrm{pH} 7.1$ and incubated at room temperature for $3 \mathrm{~h}$. The reaction was terminated by adding $1 \mathrm{mM}$ 2-mercaptoethane sulphonate sodium (MESNA). The protein was separated from the unreacted dye on a desalting column (GE Healthcare) equilibrated in the exchange solution without TFP and stored at $-80^{\circ} \mathrm{C}$ freeze-dried or in solution. The labelling efficiency was determined by spectrophotometry using the extinction coefficients of the particular dye (AlexaFluor488, $\varepsilon_{488}=71,000 \mathrm{M}^{-1} \mathrm{~cm}^{-1}$; AlexaFluor594, $\varepsilon_{594}=73,000 \mathrm{M}^{-1} \mathrm{~cm}^{-1}$; 5-IAF, $\varepsilon_{488}=88,000 \mathrm{M}^{-1} \mathrm{~cm}^{-1}$ ), calculating the protein concentration as $C_{\mathrm{ELC}}=\mathrm{A} 280$ A594/R, where $R$ is the ratio of Alexa594 absorption at 594 and $280 \mathrm{~nm}$, as an example. Typically, 60-80\% of the ELC was labelled.

Muscle fibre preparation

Muscle fibres were harvested from adult New Zealand White rabbits, killed in accordance with the Code of Practice for the Humane Killing of Animals under Schedule 1 of the Animals (Scientific Procedures) Act 1986 (UK). Small bundles of muscle fibres were dissected from the psoas muscle and permeabilised as described previously (Thirlwell et al. 1994). Fibre bundles were stored at $-20^{\circ} \mathrm{C}$ in relaxing solution containing $50 \%$ glycerol and used for experiments over a period of up to 8 weeks. Single muscle fibres, 4-5 mm long, were isolated in relaxing solution on a cooled microscope platform. Aluminium T-clips (Photo Fabrication Services Ltd., Cambridge, UK) were crimped onto each end of the fibre (Goldman and Simmons 1994), allowing the mounting of the fibre on the experimental setup. The fibres were demembranised in relaxing solution containing $1 \%$ Triton X-100 and used for ELC exchange immediately after washing with relaxing solution. The single permeabilised fibres were suspended between two hooks in a chamber, with one of the fibre ends attached to a force transducer (AE801 Sensor One Technologies, CA, USA) and the other end attached to a micrometer drive (Mitutoyo). The sarcomere length was adjusted to $2.4 \mu \mathrm{m}$ using the diffraction pattern generated by illuminating a section of the fibre with a 532-nm laser diode.

\section{ELC exchange}

The exchange solution containing $2 \mathrm{mg} / \mathrm{ml}$ labelled ELC was injected into the chamber, and the temperature was raised to $37^{\circ} \mathrm{C}$ for $30 \mathrm{~min}$ (Borejdo et al. 2001). After the incubation, the temperature was lowered to $15^{\circ} \mathrm{C}$, the fibre was extensively washed in relaxing solution, and further incubated for $30 \mathrm{~min}$ at $15^{\circ} \mathrm{C}$ in relaxing solution containing $1 \mathrm{mg} / \mathrm{ml}$ Troponin $\mathrm{C}$ (extracted from rabbit psoas muscle). To estimate the efficiency of ELC exchange, the fibre protein composition was analysed using $8 \%$ sodium dodecyl sulphate polyacrylamide gel electrophoresis (SDS-PAGE). From an isolated ELC used for exchange, a ratio of fluorescence and Coomassie signals was found. Then, from the band corresponding to the ELC in the treated muscle fibre, the proportion of the exchanged ELC was found as the difference between the isolated and the treated fibre ELC staining ratios. Based on these calculations, about $60-70 \%$ of the native ELC was exchanged with recombinant protein. The confocal images (see Figs. 2, 3 and Online Resource ESM4) show that the labelling is confined to the A-band. 


\section{SH1 labelling}

The single permeabilised fibres were incubated for $15 \mathrm{~min}$ in a relaxing solution containing $5 \mu \mathrm{M}$ AlexaFluor 488 maleimide, at $25^{\circ} \mathrm{C}$. After the incubation, the fibre was washed several times in relaxing solution to remove free dye from the fibre matrix (Berger et al. 1996; Tanner et al. 1992). As for the ELC exchange, the efficiency of labelling was estimated by SDS-PAGE. Fluorescent molecules were carried by $80 \%$ of SH1. The confocal images (see Figs. 2, 3 and Online Resource ESM4) show that the labelling is confined to the A-band.

Fluorescently labelled nucleotide analogue

The fluorescent ATP analog, $3^{\prime}-O-\{N-[3$-(7-diethylaminocoumarin-3-carboxamido)propyl]carbamoyl \}ATP (DEACpda-ATP), is a substrate for skeletal actomyosin ATPase (for simplicity, the abbreviation DEAC-ATP will be used hereinafter). DEAC-ATP shows rate constants for association and dissociation with myosin S1 that are comparable to those found for ATP. The fluorescent ATP analogy was synthesised as described in Webb et al. 2004 (extinction coefficient $46,800 \mathrm{M}^{-1} \mathrm{~cm}^{-1}$ at $430 \mathrm{~nm}$ ). The single permeabilised fibres were kept in a rigor solution containing $10 \mu \mathrm{M}$ DEAC-ATP and no other nucleotide in the absence of BDM. In this solution, the fibre was essentially in rigor, and actomyosin crossbridges formed in the overlap region (García et al. 2007). The confocal images show that labelling is confined to the A-band. Lifetime images revealed that the fluorophore is present in two populations: free in solution and bound to the actomyosin complex, with abundance of $40 \%$ and $60 \%$, respectively.

\section{Actin labelling}

The single permeabilised fibres were incubated for $45 \mathrm{~min}$ in relaxing solution containing $5 \mu \mathrm{M}$ rhodamine phalloidin, at $25^{\circ} \mathrm{C}$. After the incubation, the fibre was washed several times in relaxing solution (Prochniewicz-Nakayama et al. 1983). The confocal images show that labelling is confined to the actin band (see Online Resource ESM5).

\section{FRET imaging}

All measurements were carried out on a Leica TCS SP5 upright microscope (Leica Microsystems Ltd, Milton Keynes, UK) equipped with a a frequency-doubled, modelocked Ti:sapphire laser (Spectra-Physics Ltd. model Mai-Tai). An HCX PL APO NA 1.20 water immersion objective was used. The sarcomere length of the fibres was adjusted in relaxing solution to $2.4 \mu \mathrm{m}$ to maximise myofilament overlap. Next, the relaxing solution was changed first to a pre-rigor solution in which the fibre was incubated for $2 \mathrm{~min}$ to reduce [ATP] in the fibre core (BDM reduces rigor tension and maintains sarcomere order) and later with the rigor solution. An average force of $20 \mathrm{kN} / \mathrm{m}^{2}$ was developed by the fibre, confirming cross-bridge attachment and the rigor state.
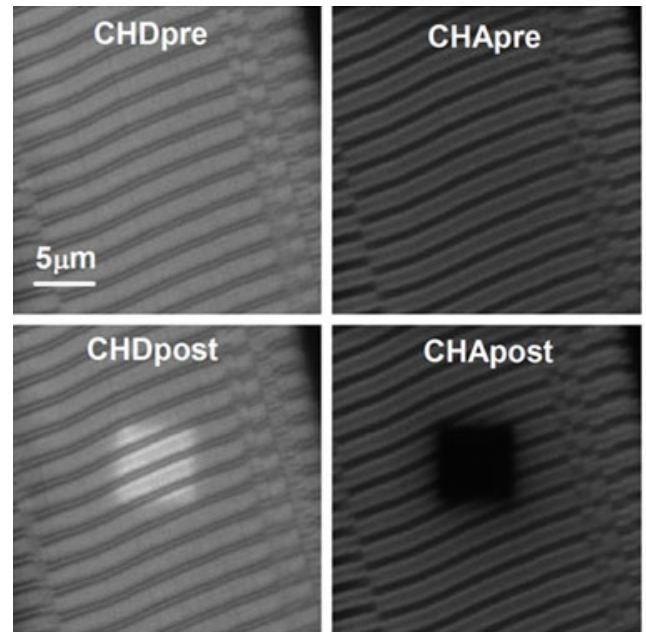

Fig. 2 An example acceptor photobleaching experiment performed to investigate the SH1-ELC interaction. In this case the images refer to a permeabilised muscle fibre of the rabbit psoas muscle at $20^{\circ} \mathrm{C}$. The banding in the sample is characteristic of the regular sarcomeric arrangement of myofilaments in striated muscle. The donor (CHD) and acceptor (CHA) fluorescence intensity is acquired before (pre) and after (post) complete acceptor photobleaching performed in the

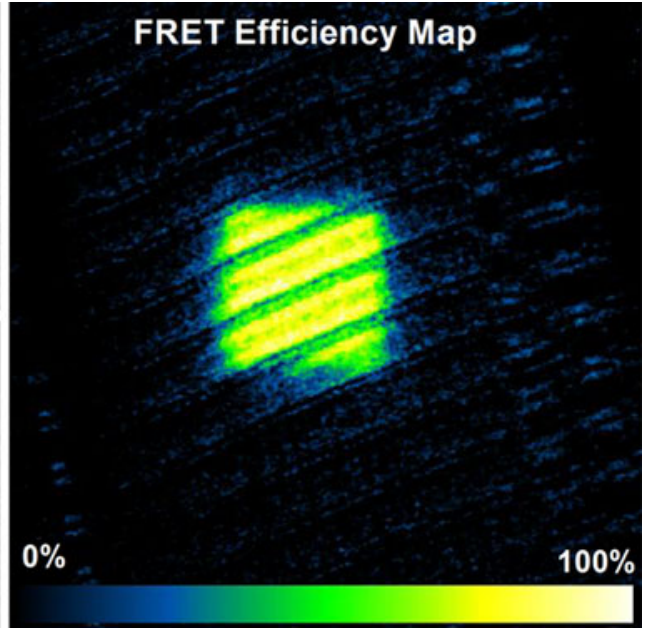

central square area. Before evaluating the FRET efficiency, images are corrected by background subtraction, and a control on a nonbleached region of equal size is performed. On the right, a map in false colours of the measured FRET efficiencies is shown. In the present experiment, an average measured FRET efficiency of $60 \%$ is evaluated (Eq. 6) 

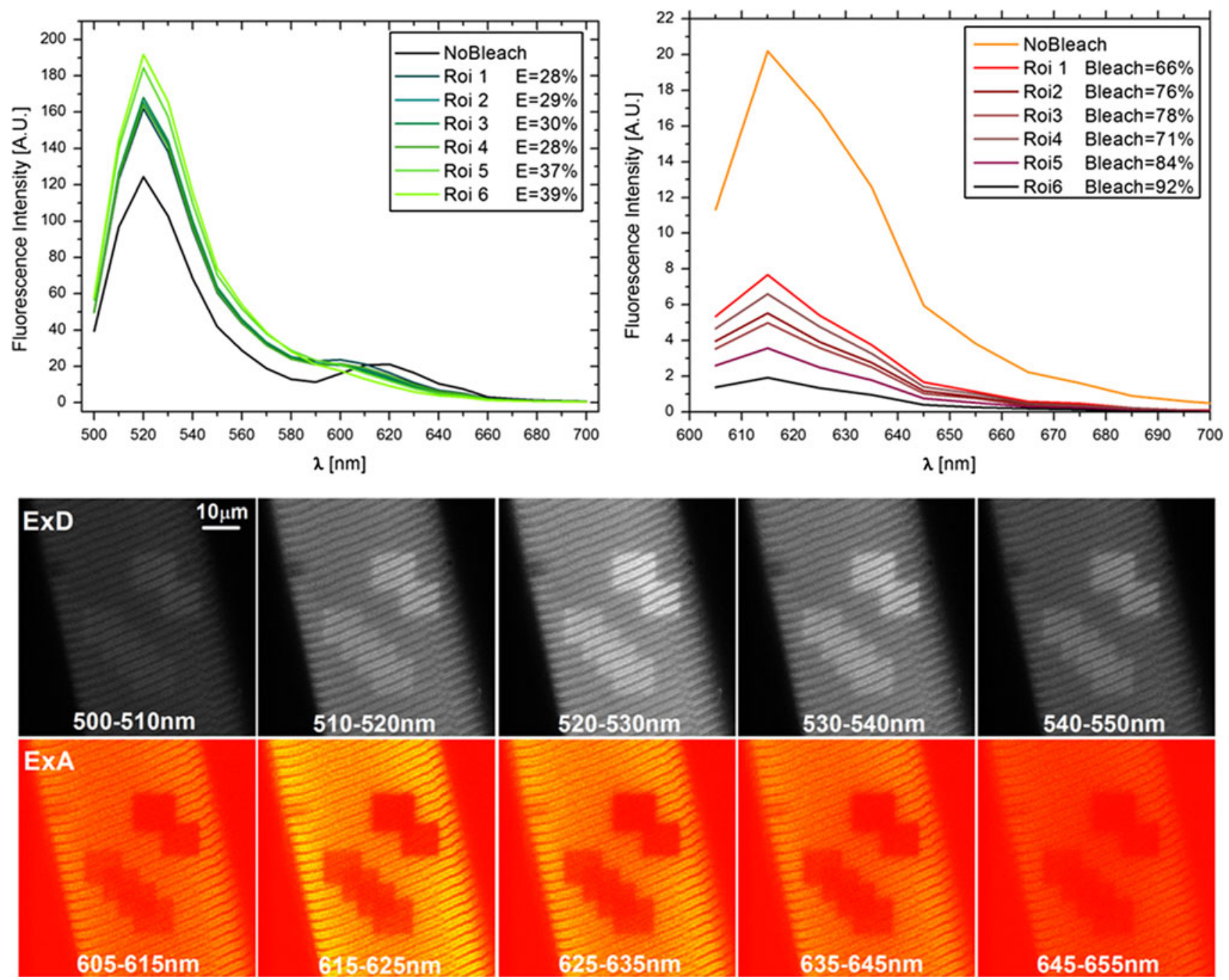

Fig. 3 Spectral analysis coupled with the acceptor photobleaching method is shown for SH1-ELC interaction. The emission spectra (exciting the donor molecule, left, and the acceptor molecules, right) have been collected for different extent of photobleaching (Bleach in the figure legend top right) to determine the optimum conditions (in terms of time and excitation power) to achieve complete acceptor photobleaching and therefore maximum donor increase ( $E$ in the top left figure legend, is the FRET efficiency evaluated at the different percentage of bleaching). The images below show the intensity collected in subsequent detection channels to acquire the spectrum (exciting the donor molecules, upper row, and the acceptor molecules, lower row)
To excite the different fluorophores at the optimum excitation wavelength, light at 488, 543, 594, 790 and $850 \mathrm{~nm}$ was provided by an argon laser $(488 \mathrm{~nm})$, two HeNe lasers (543 and $594 \mathrm{~nm}$ ) and a Ti:sapphire laser (790 and $850 \mathrm{~nm}$ ). The donor and acceptor detection channels were chosen to minimise spectral contaminations (Table 2). The excitation power was adjusted for each line to have an average power of $10 \mu \mathrm{W}$ on the sample. Images were acquired in a format of $512 \times 512$ pixels with frequency of 400 lines per second ( 0.78 frame/s).

Emission spectra were acquired for DEAC by collecting fluorescence signal from 400 to $600 \mathrm{~nm}(10 \mathrm{~nm}$ bandwidth), for both Alexa488 and 5-IAF from 500 to $700 \mathrm{~nm}$ (10 $\mathrm{nm}$ bandwidth), for rhodamine $555-705 \mathrm{~nm}(10 \mathrm{~nm}$ bandwidth) and for Alexa594 605-705 nm (10 nm bandwidth). Images were acquired in a format of $512 \times 512$ pixels with frequency of 1,000 lines per second to avoid photobleaching during the collection time (1.95 frame/s).

Acceptor photobleaching was achieved by illuminating the acceptor molecules with high excitation power $(30 \mu \mathrm{W}$ on the sample area): $488 \mathrm{~nm}$ for IAF (time required for complete bleaching, $1 \mathrm{~min}), 543 \mathrm{~nm}$ for rhodamine (2 min) and $594 \mathrm{~nm}$ for Alexa594 (5 min). The differences in bleaching time are due to the different fluorophore photostability. For each FRET couple, control measurements were performed on donor-alone samples to check possible donor photobleaching during the acceptor photobleaching; none of the donors showed significant bleaching. 
Table 2 Excitation (ExD, ExA for the donor and acceptor, respectively) and detection channel (CHD, CHA for the donor and acceptor, respectively) optimised for each FRET couple

\begin{tabular}{llllllr}
\hline FRET couple & $E x D(1 \mathrm{P})$ & $E x A(1 \mathrm{P})$ & $E x D(2 \mathrm{P})$ & ExA (2P) & CHD & CHA \\
\hline DEAC-IAF & & 488 & 850 & 790 & $430-470$ \\
Alexa488-Alexa594 & 488 & 594 & 790 & 850 & $500-540$ \\
Alexa488-rhodamine & 488 & 543 & 790 & 850 & $500-530$ \\
\hline
\end{tabular}

Both single-photon (1P) and two-photon (2P) excitation wavelengths were used. Only for DEAC-ATP, a single-photon excitation could not be used as the proper excitation laser line was not available. The reported values are in nanometres. The power output was adjusted for each excitation wavelength to have an average power of $10 \mu \mathrm{W}$ on the sample

Fluorescence lifetime imaging (FLIM) was implemented in the time domain using a Becker\&Hickl time-correlated single-photon counting (TCSPC) module (SPC-730; Becker and Hickl GmbH, Germany) coupled with the Ti:sapphire laser delivering 120-fs pulses at repetition rate of $80 \mathrm{MHz}$ (Becker 2008). To acquire a lifetime image of $256 \times 256$ pixels with 256 time bins, an acquisition time of 2 min was typically used.

\section{Characterisation of FRET couples}

Excitation and emission spectra were measured on single muscle fibres in rigor conditions to fully characterise the fluorescent properties of both donor and acceptor molecules within the biological environment. For control measurements, fibres labelled with only donor molecules or only acceptor molecules were analysed. Excitation and emission spectra were normalised to represent the probability of excitation and emission, respectively: the integral over the entire spectrum was normalised to a value of 1 , and then weighted by the extinction coefficient or the quantum yield, respectively (Online Resource ESM1). Excitation and emission spectral analysis was used to evaluate the experimental Förster radius $\left(R_{0}\right.$, Eq. 4$)$, the donor-acceptor distance for which 50\% FRET efficiency is expected. This parameter fully characterises the FRET couple (Stryer 1978):

$R_{0}=9.73 \AA\left(\frac{Q_{\mathrm{D}} \kappa^{2}}{n^{4}} J\right)^{1 / 6}$

where $Q_{\mathrm{D}}$ is the donor quantum yield, $\kappa^{2}$ is the orientation factor (equal to $2 / 3$ for freely rotating molecules, Dale et al. 1979), $n$ is the refractive index and $J$, the spectral overlap integral, is given by

$J=\frac{\int F_{\mathrm{D}}(\lambda) \varepsilon_{\mathrm{A}}(\lambda) \lambda^{4} \mathrm{~d} \lambda}{\int F_{\mathrm{D}}(\lambda) \mathrm{d} \lambda}$,

with $F_{\mathrm{D}}$ the donor emission spectrum and $\lambda$ the wavelength. It is important to characterise the fluorophores spectral behaviour in the fibre environment, as this could affect both the excitation and the emission, leading to
Table 3 Experimental and theoretical Förster radii $\left(\operatorname{Exp} R_{0}\right.$ and Theo $R_{0}$, in $\AA$ ) evaluated by Eq. 4 for the FRET couples used

\begin{tabular}{lll}
\hline FRET couple & Exp $R_{0}(\AA)$ & Theo $R_{0}(\AA)$ \\
\hline DEAC-IAF & 56.11 & 51.03 \\
Alexa488-Alexa594 & 65.69 & 65.77 \\
Alexa488-rhodamine & 71 & 71
\end{tabular}

The experimental values were evaluated by using the spectra measured directly on single-skinned muscle fibres. The theoretical values were evaluated by using the theoretical spectra (provided by Invitrogen)

Förster radius calculations that are very different from the theoretical ones (reported in Table 3, see also Online Resource ESM1). Furthermore, experimental excitation and emission spectra were critically important to evaluate the spectral contaminations that affect FRET measurements (Chen et al. 2007), namely donor emission in the acceptor channel, i.e. donor cross-talk (DCT), and direct excitation of the acceptor by the donor excitation wavelength, i.e. acceptor spectral bleed-through (ASBT) (see also Online Resources ESM2 and ESM3).

\section{FRET methods}

Experimentally, the amount of energy transfer can be evaluated by observing fluorescence variations: when FRET occurs, the fluorescence emission of the donor molecule decreases (quenching), and that of the acceptor increases. Many algorithms have been developed to calculate FRET, either by observing quenching of the donor emission or enhancement of the acceptor signal. The method of choice generally depends on the sample characteristics such as structure, morphology and function (Berney and Danuser 2003). Here, different approaches were used depending on the FRET couple analysed. For SH1-ELC and actin-ELC interactions, as the fluorophores are fixed in precise positions within the muscle fibre, FRET was quantified by observing induced donor fluorescence variations, coupling both acceptor photobleaching method and spectral analysis. By contrast, to characterise DEACATP-ELC interaction, acceptor fluorescence variations 
were followed using spectral analysis only. This approach was used because, in the DEAC-ATP-ELC sample, the fluorescently labeled ATP analogue (donor molecule) is present in at least two populations: bound to the actomyosin complex and free in solution. The free population of ATP results in a worse signal-to-noise ratio in the donor acquisition channel, which can mask the small donor fluorescence variations to be observed. Therefore for the DEAC-ATP-ELC couple it was simpler to follow the fluorescence variations in the acceptor channel, a signal which arises from molecules fixed in a precise position (ELC). In all cases, lifetime analysis was performed as an independent control of the single FRET couple efficiency. Indeed, lifetime is not affected by fluorophore concentration, so that the efficiency of FRET for the single couple and the fraction of interacting and non-interacting donors can be determined.

\section{Acceptor photobleaching}

For SH1-ELC and actin-ELC interactions, both the donor and the acceptor molecules are bound in precise positions within the muscle fibre. FRET was characterised in steadystate conditions by means of the acceptor photobleaching method. In this method, as shown in Fig. 2, the steady state is perturbed by photo-destruction of the acceptor molecules; in the absence of acceptor, the intensity of donor fluorescence which has been quenched by the acceptor increases back to its un-quenched level (Kenworthy 2001). To quantify the FRET efficiency, the donor fluorescence

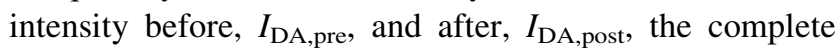
acceptor photobleaching are compared:

$\langle E\rangle_{\mathrm{D}}=1-\frac{\left\langle I_{\mathrm{DA}, \text { pre }}\right\rangle-B_{\mathrm{DA}, \text { pre }}}{\left\langle I_{\mathrm{DA}, \text { post }}\right\rangle-B_{\mathrm{DA}, \text { post }}}$,

where $B_{\mathrm{DA} \text {,pre }}$ and $B_{\mathrm{DA} \text {,post }}$ are the background detected in the donor acquisition channel before and after complete acceptor photobleaching. The present measurements are carried out with particular attention to complete photobleaching of the acceptor to avoid any underestimation of FRET due to partial bleaching. Moreover, to exclude that the observed donor intensity variations are associated with any other processes which are not FRET (for example donor photobleaching during image acquisition, or focus changes), the donor fluorescence intensity was monitored not only in the acceptor photobleaching region but in other parts of the sample where bleaching was not performed. For each FRET couple, control measurements were performed on donor-alone samples to check possible donor photobleaching during the acceptor photobleaching. None of the donors showed significant bleaching (Online Resource ESM4). Furthermore, it is important to couple the acceptor photobleaching method with spectral analysis, as the latter can reveal changes or shifts in the spectral emission properties which can signal the existence of processes other than FRET in the sample (photo-conversion, changes in $\mathrm{pH}$ etc.).

\section{Spectral analysis}

Spectral analysis was performed for SH1-ELC and actinELC interactions to confirm that the fluorescence variations observed in the acceptor photobleaching experiments are associated with FRET and are not caused by other phenomena affecting the spectral properties of the interacting molecules. Emission spectra exciting sequentially the donor molecules and the acceptor molecules were acquired before and after acceptor photobleaching to check that spectral shape changes or wavelength shifts did not occur for either molecule.

The fluorescence emission spectrum for the donor (Fig. 3, left) shows two peaks, one centred on the donor emission peak and the second on the acceptor emission peak. The latter in general can be due to several contributions: FRET signal, direct excitation of the acceptor molecules by the donor excitation wavelength (ASBT, acceptor spectral bleed-through) and donor emission in the acceptor emission range of wavelengths (DCT, donor cross-talk). By exciting the acceptor molecules, the characteristic emission of the acceptor is observed (Fig. 3, right). After complete photobleaching of the acceptor, the peak corresponding to the acceptor emission disappears, and an increase in the donor emission peak is obtained. Since no wavelength shifts and no shape variations are observed, the donor fluorescence variations are attributed to FRET.

For the DEAC-ATP-ELC couple, a different approach was followed, mainly observing the acceptor fluorescence variations: firstly, a fibre labelled with only the acceptor molecules was characterised in terms of its spectral properties, with particular attention to estimation of the direct acceptor excitation at the donor excitation wavelength (Fig. 4, left panel, orange curve). Secondly, DEAC-ATP was added to the solution, and consequent fluorescence variations were followed (dark green curve). FRET efficiency is deduced by applying a spectral unmixing algorithm to the fluorescence spectrum of the complex (Zimmermann 2005; Nashmi et al. 2005; Thaler et al. 2005), which provides an estimate of the apparent donor contribution and of the apparent acceptor contribution (light green and red curves, respectively). Since the red curve represents FRET plus ASBT contributions, and the orange curve represents the ASBT in the very same sample, thus the FRET efficiency can be easily calculated. On the right, a very similar experiment is shown, but in this case, instead of adding DEAC-ATP, free coumarin is added to 


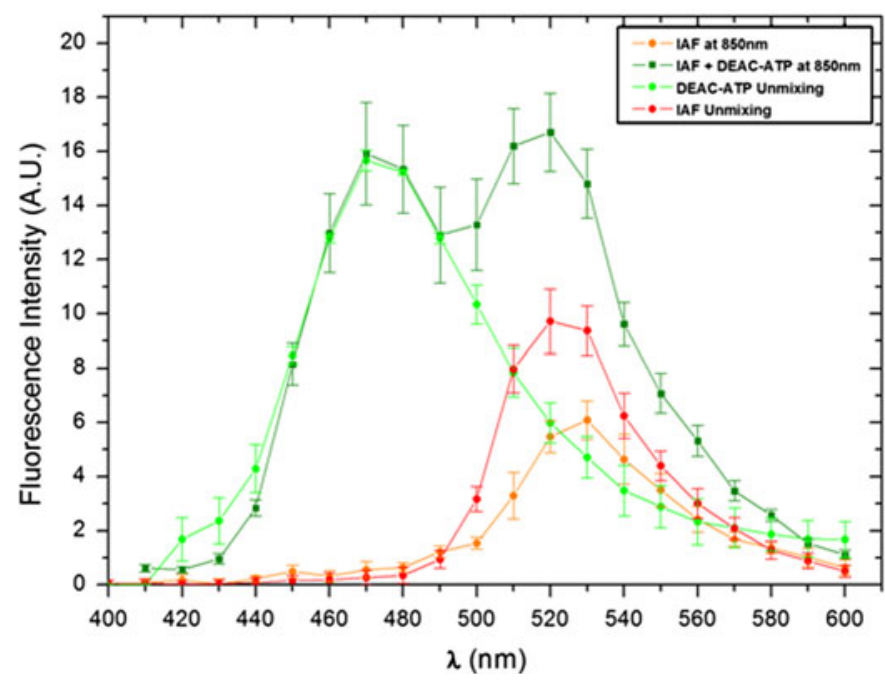

Fig. 4 Spectral analysis for characterising DEAC-ATP-ELC interaction (left panel). Acceptor-alone signal exciting at the donor excitation wavelength (orange curve, ASBT contribution); spectrum of the complex (dark green curve). Apparent donor contribution and

the solution. The coumarin free in solution does not specifically bind to myosin in the nucleotide binding pocket as occurs for DEAC-ATP, thus providing a control measurement on the detected FRET; as can be observed in this case, the red and orange curves are similar, showing that FRET is not occurring.

\section{Lifetime analysis}

The donor fluorescence lifetime in the presence of an acceptor is shortened; therefore, the occurrence of FRET can be measured by monitoring the change in donor lifetime in the presence and absence of the acceptor (Chen et al. 2005):

$\langle E\rangle_{\mathrm{D}}=1-\frac{\tau_{\mathrm{DA}}}{\tau_{\mathrm{D}}}$.

As a result, lifetime-based FRET approaches have significant advantages over intensity-based FRET approaches. As only the donor lifetime is monitored, no DCT or ASBT correction is needed, and stoichiometry information can be gained (Wallrabe and Periasamy 2005). In addition, the measured FRET efficiency is equal to the true FRET efficiency of a single FRET couple, as lifetime measurements are not affected by concentrations:

$\langle E\rangle_{\mathrm{D}}=E_{\text {single }}$.

In Fig. 5, a first FRET-FLIM analysis is shown. The photon counts are imaged in Fig. 5a for a donor-acceptor sample and Fig. $5 \mathrm{~b}$ for a donor-alone sample. FLIM-FRET measurements are optimised in terms of signal-to-noise ratio considering that the sarcomeres in the muscle fibre

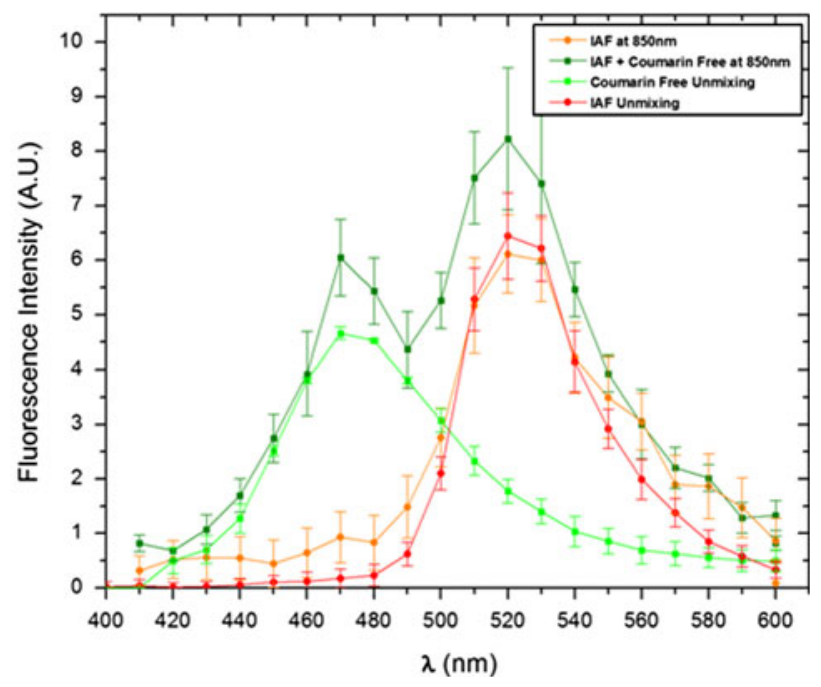

apparent acceptor contribution estimated by spectral unmixing algorithm (light green and red curves, respectively). The same spectral analysis for free coumarin and ELC interaction (right panel), showing that FRET is not occurring

consist of A- and I-bands: the A-bands contain the myosin molecules, and parts of the actin filaments, while the I-bands contain actin filaments that are not in the filament overlap zone. The signal-to-noise ratio in the lifetime maps is improved by masking off the I-bands (Fig. 5c and Fig. 5d), thus only photons from the A-band were considered in the analysis. Each pixel within the mask is fitted with a single exponential decay, and the related average lifetime value is imaged in Fig. 5e and Fig. 5f, showing that the donor-acceptor sample presents a shorter lifetime, on average, as is clearly revealed by the lifetime distribution in Fig. $5 \mathrm{~g}$.

To evaluate the FRET efficiency, for SH1-ELC interaction, a double exponential fit is carried out for the donoracceptor sample, as both interacting and non-interacting donors contribute to the measured decay function, presenting a fast and a slow lifetime component, respectively (Fig. 6, left, empty square dots and light grey line). In this case, just one measurement is necessary to evaluate the FRET efficiency as, from the double exponential fit, $\tau_{\mathrm{D}}$ and $\tau_{\mathrm{DA}}$ can be directly extrapolated:

$I(t)=\left(n_{\mathrm{D}}-m_{\mathrm{D}}\right) e^{-\frac{t}{\tau_{\mathrm{D}}}}+m_{\mathrm{D}} e^{-\frac{t}{\tau_{\mathrm{DA}}}}$.

with $n_{\mathrm{D}}$ and $m_{\mathrm{D}}$ defined as in Eqs. 1 and 2. The donoralone sample was fitted with a single exponential, and the extracted lifetime was used as control on the longer component evaluated for the donor-acceptor sample (Fig. 6, left, filled black square dots and dark grey line). For DEAC-ATP-ELC interaction, a double exponential fit was necessary in the donor-alone sample as free DEAC-ATP in solution had to be taken into account (Fig. 6, middle, filled black square dots and dark grey line). The donor-acceptor 

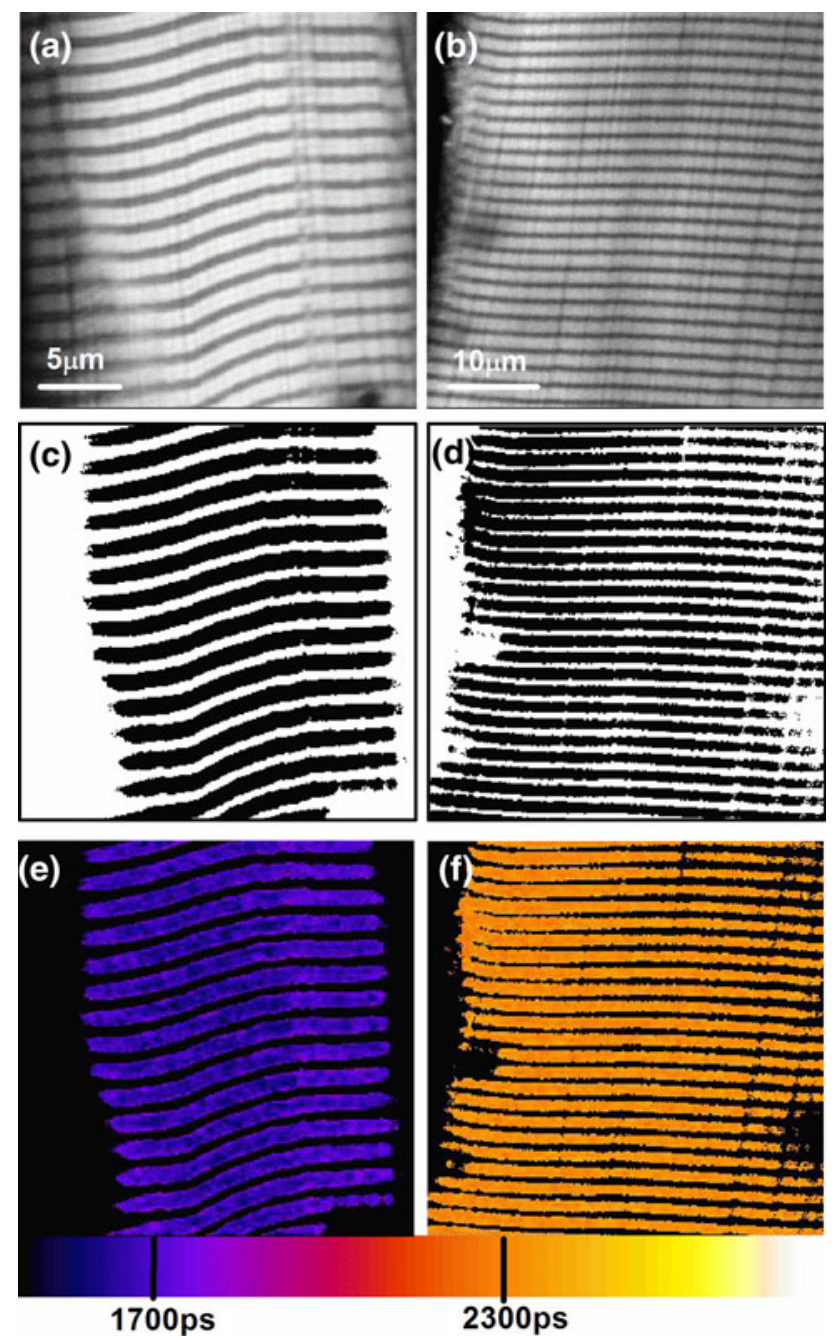

$1700 \mathrm{ps}$

2300ps

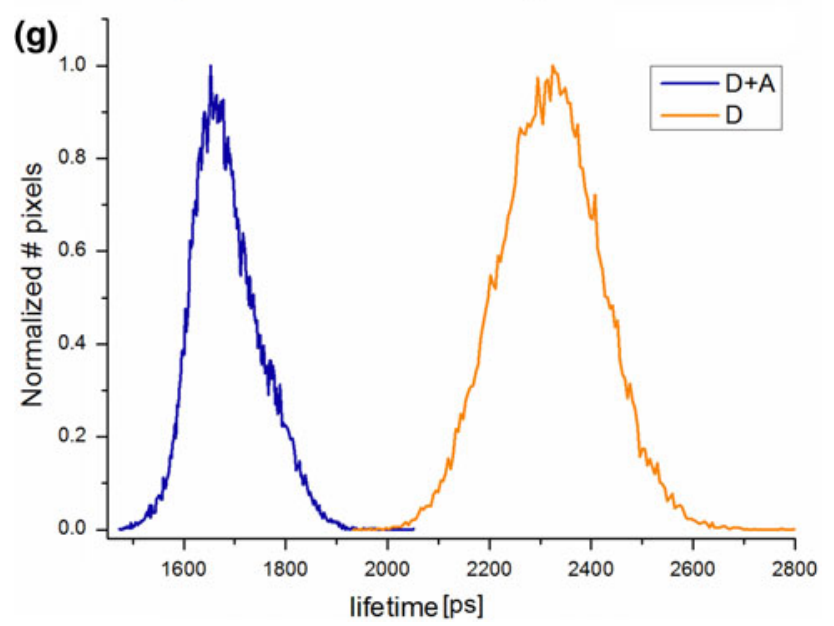

Fig. 5 Lifetime analysis performed to study the SH1-ELC interaction: left column refers to a fibre labelled with both donor and acceptor molecules, right column for a donor-alone sample. Photon count images (a) and (b); mask superimposed to isolate only the A-band photons (c) and (d); colour-coded lifetime map (e) and (f); lifetime distribution obtained by fitting each pixel with a single exponential decay $(\mathbf{g})$ sample was therefore fitted with a triple exponential fit, assigning the fastest component $\left(\tau_{\text {Free }}\right)$ to the lifetime value of the free DEAC-ATP component obtained from the donor-alone sample (Fig. 6, middle empty square dots and light grey line):

$I(t)=\left(n_{\mathrm{D}}-m_{\mathrm{D}}-m_{\text {Free }}\right) e^{-\frac{t}{\tau_{\mathrm{D}}}}+m_{\mathrm{D}} e^{-\frac{t}{\tau_{\mathrm{DA}}}}+m_{\text {Free }} e^{-\frac{t}{\tau_{\text {Free }}}}$.

For the ELC-actin interaction, a single exponential fit was the best fit for both the donor-alone sample and the donor-acceptor sample, demonstrating that FRET was not occurring (Fig. 6, left).

\section{Results and discussion}

All the FRET experiments described herein were performed on rigor muscle fibres: in this condition, most of the myosin heads are attached to actin, with myosin expected to be in an open configuration (Yang et al. 2007). The number of attached cross-bridges depends on the extent of the overlap region. At sarcomere length of $2.4 \mu \mathrm{m}$, we expect the number of attached heads to exceed $80 \%$ (Higuchi et al. 1995). For the couple DEAC-ATP-ELC, the term "rigor condition" is used for simplicity; it is worth underlying that, in the presence of a low concentration $(10 \mu \mathrm{M})$ of fluorescently labelled ATP in the solution, the fibre remains in a rigor-like state, in particular in an adenosine diphosphate (ADP)-bound state (García et al. 2007). The choice of the three FRET couples here analysed is linked to our interest in the ELC C-terminus, which forms an interface with the catalytic domain. For this reason we labelled ELC-180 and observed its interaction with three different regions in the actomyosin complex; we chose to investigate the energy transfer with the ATP analogue and with SH1 to show the capability to distinguish small FRET variations (the theoretical D-A distance is nearly 4 and $5 \mathrm{~nm}$, respectively) and the interaction with actin as negative FRET control (theoretical D-A distance of nearly $10 \mathrm{~nm}$, too large to reveal energy transfer).

For SH1-ELC and actin-ELC interactions, both acceptor photobleaching measurements (Fig. 2) and spectral analysis (Fig. 3) were carried out, observing donor fluorescence variations, while for DEAC-ATP-ELC acceptor, fluorescence variations were followed only by spectral analysis (as described in the "FRET methods" section; see Fig. 4). For all three couples, spectral analysis was essential to ascertain that the observed fluorescence variations were due to FRET and not to other phenomena affecting the spectral properties of the interacting molecules, as clearly shown in Figs. 3 and 4. The measured FRET efficiencies $(\langle E\rangle)$ from intensity-based measurements for the 

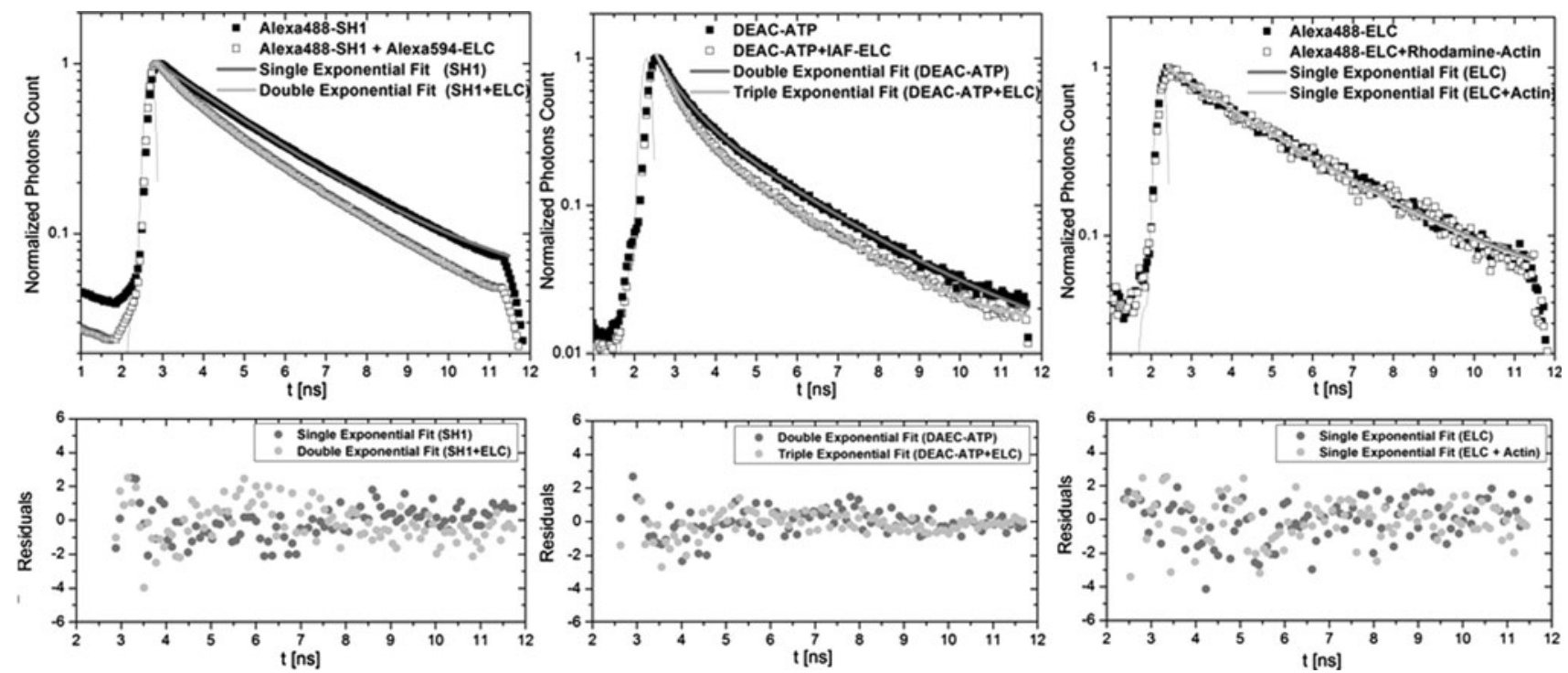

Fig. 6 Lifetime decay curves for the three FRET couples: donoralone samples (filled black square), donor-acceptor samples (empty black square), fit for donor alone (dark grey line) and fit for donoracceptor (light grey line). For DEAC-ATP alone, a double exponential fit was required due to the presence of free fluorophore in solution, and a triple exponential fit when adding the acceptor; for both ELC-

SH1 and ELC-actin, a single exponential fit was sufficient for the donor-alone decay curve, with a double exponential fit when adding the acceptor for the ELC-SH1 interaction, and a single again for ELC-actin, confirming the negative FRET control. Bottom panels show the standardised residuals (residuals divided by their estimated standard error) for each fit

Table 4 Measured FRET efficiencies $(\langle E\rangle)$ evaluated by intensity-based methods (both acceptor photobleaching and spectral analysis gave similar results)

\begin{tabular}{|c|c|c|c|c|}
\hline FRET couple & $\langle E\rangle(\%)$ & $m_{\mathrm{D}}=m_{\mathrm{A}}(\%)$ & $E_{\text {single }}(\%)$ & $r(\AA)$ \\
\hline Alexa488 SH1-Alexa594 ELC & $57 \pm 4(n=15)$ & 60 & $95 \pm 6$ & $40 \pm 6$ \\
\hline DEAC ATP-IAF ELC & $40 \pm 4(n=25)$ & 30 & $61 \pm 5$ & $52 \pm 5$ \\
\hline Alexa488 ELC-phalloidin actin & $0 \pm 5(n=8)$ & 0 & 0 & $>100$ \\
\hline
\end{tabular}

$n$ is the number of fibres analysed for each couple. For each fibre, many FRET experiments were carried out to evaluate the average measured FRET efficiency presented in the table; $m_{\mathrm{D}}$ is the fraction of interacting donor estimated by SDS-PAGE, only for DEAC ATP-IAF ELC couple it was estimated by lifetime; single FRET efficiencies $\left(E_{\text {single }}\right)$ evaluated by Eq. 1, 2 and the related D-A distances $(r$, in $\AA$ ) calculated by Eq. 3

different pairs are reported in Table 4. The FRET efficiency values are the average measured FRET efficiencies determined for $n$ fibres per experiment. More specifically, the measured FRET efficiency values for the ELC-SH1 (Table 4, first row) and ELC-actin (Table 4, last row) interactions refer to average values obtained by applying the acceptor photobleaching method, while the measured FRET efficiency for the ELC-DEAC-ATP interaction (Table 4, middle row) refers to the average value obtained by applying spectral analysis and unmixing algorithm (see "Materials and methods" section). It is worth noting the small difference between the two measured FRET efficiencies for ELC-SH1 and ELC-DEAC-ATP and the expected zero FRET efficiency evaluated for the ELCactin interaction. The number of donors, $n_{\mathrm{D}}$, and the fraction of interacting donors, $m_{\mathrm{D}}$ (or $n_{\mathrm{A}}$ and $m_{\mathrm{A}}$, respectively, for the acceptor), reported in Table 4 , were estimated by taking into consideration the percentage of labelling evaluated by SDS-PAGE gel for the different labelled positions (as explained in the "Materials and methods" section). For DEAC-ATP, only lifetime analysis was used to evaluate these interacting/non-interacting fractions due to the reversibility of nucleotide binding.

From Eqs. 1 and 2 the true FRET efficiencies (Table 4) for a single interacting D-A couple $\left(E_{\text {single }}\right)$ and the related D-A distances (Eq. 3) were evaluated. Due to the fraction of non-interacting donors, the measured FRET efficiencies in the first two cases are underestimates of the true values. This highlights the importance of assumptions when calculating the donor-acceptor distances. The zero measured FRET efficiency found for the third interaction represents the sensitivity of our measurements (less than $10 \%$ on $\langle E\rangle$ ).

Direct evaluation of FRET efficiency was achieved from the lifetime measurements; the measured FRET efficiency is equal to the single FRET efficiency (Eq. 8), as lifetime is not affected by concentration. The measured FRET 
Table 5 Single FRET efficiency ( $\left.E_{\text {single }}\right)$ evaluated by lifetime measurements

\begin{tabular}{llllll}
\hline FRET couple & $m_{\mathrm{D}}(\%)$ & $\tau_{\mathrm{F}}(\mathrm{ns})$ & $\left(n_{\mathrm{D}}-m_{\mathrm{D}}\right)(\%)$ & $\tau_{\mathrm{D}}(\mathrm{ns})$ & $2.6 \pm 0.1$ \\
\hline Alexa488 SH1-Alexa594 ELC & $60 \pm 2$ & $0.4 \pm 0.1$ & $40 \pm 2$ & $70 \pm 2$ & $2.3 \pm 0.1$ \\
DEAC ATP-IAF ELC & $30 \pm 2$ & $0.7 \pm 0.1$ & 100 & $2.8 \pm 0.2$ \\
Alexa488 ELC-phalloidin actin & 0 & 0 & $0.6 \pm 5$ \\
\hline
\end{tabular}

$n_{\mathrm{D}}$ is the number of donors, $m_{\mathrm{D}}$ is the fraction of interacting donors; $\tau_{\mathrm{F}}$ and $\tau_{\mathrm{D}}$ are the FRET and the donor lifetime component: the fast and the slow component from the double exponential fit, respectively, for SH1-ELC and actin-ELC interactions; for DEAC-ATP-ELC, a triple exponential was used as described in the "FRET methods" section, but for simplicity here only the donor and the FRET component are reported (the fastest component of the free ATP in solution showed a lifetime of $0.5 \mathrm{~ns}$ with abundance of $40 \%$ ). $E_{\text {single }}$ was evaluated as described in Eqs. 7 and 8

efficiencies for the three FRET couples are reported in Table 5. The values for both $m_{\mathrm{D}}\left(m_{\mathrm{D}}=m_{\mathrm{A}}\right)$ and $E_{\text {single }}$ evaluated from the lifetime measurements are in agreement with $m_{\mathrm{D}}$ and $E_{\text {single }}$ extrapolated from the intensity-based measurements corrected for the unpaired donors. The two approaches, i.e. intensity- and lifetime-based methods, were essential to quantify the FRET efficiency with high accuracy. In particular, lifetime analysis was crucial to confirm the validity of intensity-based measurements, so that the true single FRET efficiency could be established.

The measured FRET efficiencies corrected for the number of interactions, $m_{\mathrm{D}}$, lead to values for the true FRET efficiencies and the D-A distances which are in agreement with expectations from the crystallographic data. In particular, from the spectral characterisation of the FRET couples and the consequent evaluation of the experimental Förster radii, it has been possible to predict the theoretical FRET efficiency dependence on the distance for a single FRET pair in the present experimental conditions (Fig. 7). From the crystallographic knowledge of the myosin head structure in open configuration of the myosin head in a rigor state (Rayment et al. 1993a; Mendelson and Morris 1997; Lorenz et al. 1993), the expected theoretical donor-acceptor distances were evaluated: For SH1-ELC interaction, the theoretical D-A distance at which the molecules should be found in rigor condition is nearly $38 \AA$ (grey dashed line), while we measured nearly $40 \AA$ A; for the nucleotide binding pocket and the ELC: $51 \AA$ A (black dash-dotted line) compared with the experimental distance of $52 \AA$; for the actin-ELC interaction, the expectation distance is nearly $95 \AA$ (light grey dots line); experimentally we were not able to observe FRET, as the interaction distance is too large for FRET to occur. This couple indeed was chosen as our negative FRET control. The theoretical expected distances were measured between the attachment points of the fluorophores. Taking into account the dimensions of the fluorophores and their mobility, we estimated the errors in these measurements to be of the order of $2-3 \AA$.

We discuss here the assumption of freely rotating molecules, which gives a value of $2 / 3$ for the orientation factor.

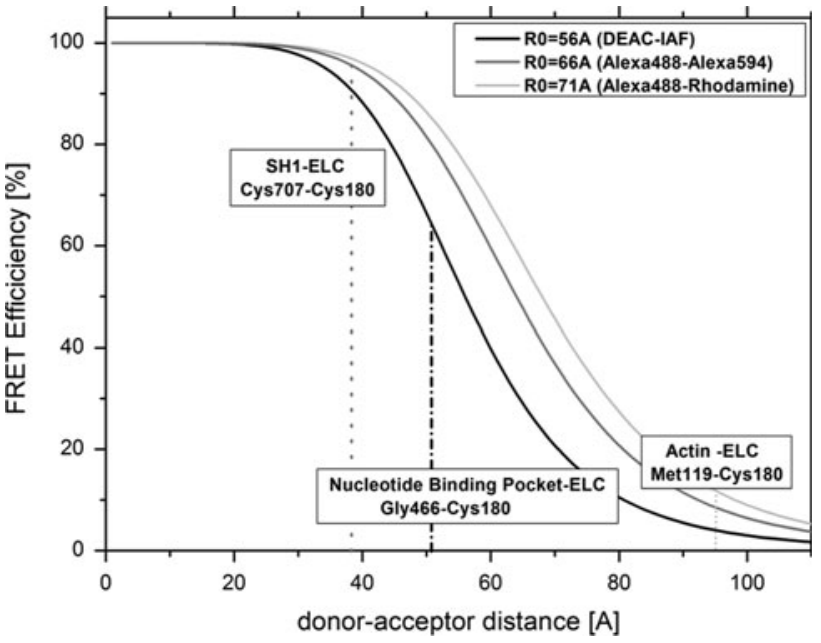

Fig. 7 Theoretical FRET efficiencies predicted for the three different FRET couples analysed: DEAC-ATP-ELC (black curve), SH1-ELC (grey curve) and actin-ELC (light grey curve). The lines represent the theoretical distances at which the molecules should be in a rigor condition as determined from the crystallographic structure: SH1ELC (grey dashed line), DEAC-ATP-ELC (black dash-dotted line) and actin-ELC (light grey dots line)

The orientation factor contributes to the evaluation of the Förster radius, which in turn has implications for the determination of the donor-acceptor distance (see Eqs. 3 and 4). The labelling procedures used for SH1 and actin may lead to loss of degrees of freedom. With regard to SH1 labelling, different groups showed limited anisotropy of fluorophores attached at this location (Ishiwata et al. 1997; Tanner et al. 1992), which was useful to probe protein rigidity/flexibility. In the case of actin, the phalloidin is located in the cleft of the actin helix, binding two or three monomers at a time, probably conferring more rigidity to the fluorophore (Lorenz et al. 1993). On the contrary, DEAC-pda-ATP and the ELC-180 labelling exhibit more flexibility: concerning DEAC-pda-ATP, the long $(\sim 1 \mathrm{~nm})$ and flexible propylenediamine (pda) linker group places the fluorophore outside the binding pocket, thus conferring flexibility (Garcia et al. 2010; Webb et al. 2004); for ELC180 , due to its location in the loops connecting the $\mathrm{G}$ and $\mathrm{H}$ helices in the C-terminal part of the ELC, we expect the 
fluorophore to exhibit some degrees of rotation (Borejedo et al. 2001). Of course anisotropy measurements are required to properly address the polarisation issue and to corroborate such hypothetical arguments. Nonetheless, the good agreement between our results and the crystallographic data suggests that the assumption of $\kappa^{2}=2 / 3$ is reasonable.

Another important aspect worth discussing concerns the possibility of inter-head FRET. As each of the two heads of a myosin molecule is probably labelled to similar extent, multiple interactions could occur and should be taken into account in the FRET theory. The measured FRET efficiency in the case of multiple acceptors should be higher than in the case of single interaction (Koushik et al. 2009). However, this additional contribution could be masked by the fraction of non-interacting donors, which instead causes an underestimation of the single FRET efficiency. Further investigations are necessary to understand how the second head could affect the measured FRET efficiency. Useful experiments could be performed by introducing a mixture of donor-labelled and acceptor-labelled ELC in the exchanging solution and by verifying the occurrence of FRET. However, the results obtained so far support the assumption that multiple interactions are not occurring.

Certainly, the agreement of the intensity-based FRET evaluations with both the lifetime analysis and the crystallographic expectations demonstrates the viability of the present approach for distinguishing small FRET variations, essential to monitor cross-bridge conformational changes, directly in active fibres.

\section{Conclusions}

FRET measurements on single permeabilised muscle fibres in the rigor state from skeletal muscle were performed to investigate molecular interactions at the cross-bridge level. In particular, three donor-acceptor pairs were studied, positioning the fluorophores at strategic locations sensitive to myosin head conformational changes. Specifically, the interactions between Cys180 on the ELC and SH1, the nucleotide binding pocket and actin were characterised. This is the first time that the method presented herein to extrapolate the true FRET efficiency and the related donoracceptor distance has been applied to study single muscle fibres. The analysis resulted in distances in agreement with the theoretical distances obtained from crystallographic data, demonstrating the viability of this new approach to reveal structural features at the cross-bridge level. This characterisation is essential before analysing cross-bridge conformational changes directly in active muscle fibres. In particular, it is crucial to demonstrate the capability of detecting FRET within the muscle fibre environment, and above all the capability of measuring small FRET changes. To this end, we investigated different FRET pairs within the cross-bridge complex. Using the same fluorescently labelled ATP analogue (DEAC-pda-ATP) we recently measured fluorescence lifetime variations as a function of strain (Garcia et al. 2010), demonstrating that the novel approach of combining simultaneous fluorescence and force records is practicable. The challenging aim of detecting distance variations under strain will be tackled by means of both intensity-based and lifetime analysis, in the first instance by applying cycles of stretch and release to rigor fibres to alter the interaction distance between the fluorophores. In this configuration, the single FRET population model described herein will still be valid. In studies of active muscle fibre, the proposed FRET model will need to be modified to take into account the different states of the cross-bridges during contraction, which, in terms of FRET, leads to consideration of multiple FRET populations and consequently to analysis of multiple distance distributions.

\section{Appendix: single FRET population case}

To evaluate the FRET efficiency $E$ from intensity-based measurements, either donor fluorescence variations or acceptor fluorescence variations can be followed. In the former case, the experimental FRET efficiency is measured by comparing the average donor fluorescence intensity detected in the donor acquisition channel in the presence $\left(\left\langle I_{\mathrm{DA}}\right\rangle\right)$ and in the absence $\left(\left\langle I_{\mathrm{D}}\right\rangle\right)$ of the acceptor (the internal subscript represents the sample: "D" for donoralone sample, "A" for acceptor-alone sample, "DA" for donor-acceptor sample), exciting at the donor excitation wavelength:

$\langle E\rangle_{\mathrm{D}}=1-\frac{\left\langle I_{\mathrm{DA}}\right\rangle_{\mathrm{D}}^{\mathrm{D}}}{\left\langle I_{\mathrm{D}}\right\rangle_{\mathrm{D}}^{\mathrm{D}}}$.

In the absence of the acceptor, the intensity detected from an ensemble of $n_{\mathrm{D}}$ donors is

$\left\langle I_{\mathrm{D}}\right\rangle_{\mathrm{D}}^{\mathrm{D}}=\varepsilon_{\mathrm{D}} n_{\mathrm{D}} I_{\mathrm{D}, \text { single }}$

where $I_{\mathrm{D} \text {,single }}$ is the fluorescence intensity detected in the donor acquisition channel which arises from a single donor particle during the acquisition time, and $\varepsilon$ is the extinction coefficient (the probability of a molecule being excited at that excitation wavelength). If $m_{\mathrm{D}}$ interactions are occurring, the fluorescence detected in the donor acquisition channel is now given by

$\left\langle I_{\mathrm{DA}}\right\rangle_{\mathrm{D}}^{\mathrm{D}}=\varepsilon_{\mathrm{D}}\left[\left(n_{\mathrm{D}}-m_{\mathrm{D}}\right) I_{\mathrm{D}, \text { single }}+m_{\mathrm{D}} E_{\text {single }} I_{\mathrm{D}, \text { single }}\right]$.

The first term represents the fraction of non-interacting donors, while the second term is the fraction of interacting 
donors. $E_{\text {single }}$ is the energy transfer efficiency for a single FRET couple, which decreases the intensity contribution of the second term. Here, all $m_{\mathrm{D}}$ interacting donors are contributing equally to FRET: all FRET couples are at identical distances and orientations, and multiple interactions are neglected (single FRET population hypothesis). Furthermore, Eq. 13 neglects the possible contribution to the fluorescence intensity of the acceptor particles directly excited by the donor excitation wavelength emitting in the donor acquisition channel, which can generally be ignored by judicious choice of fluorophores and excitation.

Substituting Eq. 12 and 13 into Eq. 11, we obtain

$\langle E\rangle_{\mathrm{D}}=\frac{m_{\mathrm{D}}}{n_{\mathrm{D}}} E_{\text {single }}$.

Equation 14 is an important relation, as it relates the average measured FRET efficiency to the true efficiency for a single interacting couple: if a fraction of the donors is not interacting (which is most likely the case), the measured FRET efficiency underestimates the true one.

Similar considerations are used to evaluate FRET by observing the acceptor fluorescence variations. In this case we take into account two major intensity contributions detected in the acceptor channel which hide the FRET signal (donor excitation, acceptor emission) due to the spectral overlap, namely donor cross-talk (DCT, donors emitting in the acceptor channel), and acceptor spectral bleed-through (ASBT, acceptors directly excited by the donor excitation wavelength). Therefore, if, in a system of $n_{\mathrm{A}}$ acceptor particles, $m_{\mathrm{A}}$ interactions are occurring $\left(m_{\mathrm{A}}=m_{\mathrm{D}}\right)$, the fluorescence detected in the acceptor acquisition channel, exciting at the donor excitation wavelength, is given by

$$
\begin{aligned}
\left\langle I_{\mathrm{DA}}\right\rangle_{\mathrm{A}}^{\mathrm{D}}= & \varepsilon_{\mathrm{D}}\left[m_{\mathrm{A}} E_{\text {single }} I_{\mathrm{A}, \text { single }}+\left(n_{\mathrm{D}}-m_{\mathrm{A}}\right) I_{\mathrm{D}, \text { single }}\right] \\
& +\varepsilon_{\mathrm{A}}\left(n_{\mathrm{A}}-m_{\mathrm{A}}\right) I_{A, \text { single }},
\end{aligned}
$$

where $\left(n_{\mathrm{D}}-m_{\mathrm{A}}\right)$ represents the fraction of non-interacting donors emitting in the acceptor channel, i.e. DCT, and $\left(n_{\mathrm{A}}-m_{\mathrm{A}}\right)$ the fraction of acceptors excited by the donor excitation wavelength, i.e. ASBT. If we excite the very same system at the acceptor excitation wavelength:

$\left\langle I_{\mathrm{DA}}\right\rangle_{\mathrm{A}}^{\mathrm{A}}=\varepsilon_{\mathrm{A}}\left[n_{\mathrm{A}} I_{A, \text { single }}\right]$.

The measured FRET efficiency in this case is evaluated by comparing the intensity of the acceptor detected exciting the donor molecules, $\left\langle I_{\mathrm{DA}}\right\rangle_{\mathrm{A}}^{\mathrm{D}}$, corrected for the DCT and ASBT, with the intensity of the acceptor detected exciting at its proper excitation wavelength, $\left\langle I_{\mathrm{DA}}\right\rangle_{\mathrm{A}}^{\mathrm{A}}$ :

$\langle E\rangle_{\mathrm{A}}=\frac{\left\langle I_{\mathrm{DA}}\right\rangle_{A_{\text {corr }}^{\mathrm{D}}}^{\mathrm{p}}}{\left\langle I_{\mathrm{DA}}\right\rangle_{\mathrm{A}}^{\mathrm{A}}}$
Substituting Eqs. 15 and 16 into Eq. 17, we obtain

$\langle E\rangle_{\mathrm{A}}=\frac{\varepsilon_{\mathrm{D}}}{\varepsilon_{\mathrm{A}}} \frac{m_{\mathrm{A}}}{n_{\mathrm{A}}} E_{\text {single }}$.

Like Eq. 14, Eq. 18 is an important relation, as it relates the average measured FRET efficiency with the true efficiency for a single interacting couple.

Acknowledgments This work was supported by the Royal Society (Newton International fellowship to V. Caorsi) and Milstein Fund, Medical Research Council (G0601747). We acknowledge the FILM facility (Facility for Imaging by Light Microscopy), M. Webb and G. Reid for providing the DEAC-pda-ATP, and N. Curtin and L. Ying for useful discussions.

Open Access This article is distributed under the terms of the Creative Commons Attribution Noncommercial License which permits any noncommercial use, distribution, and reproduction in any medium, provided the original author(s) and source are credited.

\section{References}

Becker W (2008) The BH TCSPC hand book. Becker \& Hickl, Berlin Becker W, Bergmann A, Haustein E, Petrasek Z, Schwille P, Biskup C, Kelbauskas L, Benndorf K, Klöcker N, Anhut T, Riemann I, König K (2006) Fluorescence lifetime images and correlation spectra obtained by multidimensional time-correlated single photon counting. Microsc Res Tech 69:186-195

Berger CL, Craik JS, Trentham DR, Corrie JET, Goldman YE (1996) Fluorescence polarization of skeletal muscle fibres labelled with rhodamine isomers on the myosin heavy chain. Biophys $\mathrm{J}$ 71:3330-3343

Berney C, Danuser G (2003) FRET or no FRET: a quantitative comparison. Biophys J 84:3992-4010

Borejdo J, Ushakov DS, Moreland R, Akopova I, Reshetnyak Y, Saraswat LD, Kamm K, Lowey S (2001) The power stroke causes changes in the orientation and mobility of the termini of essential light chain 1 of myosin. Biochem 40(13):3796-3803

Botts J, Takashi R, Torgerson P, Hozumi T, Muhlrad A, Mornet D, Morales MF (1984) On the mechanism of energy transduction in myosin subfragment 1. Proc Natl Acad Sci 81:2060-2064

Chen Y, Elangovan M, Periasamy A (2005) FRET data analysis: the algorithm. In: Periasamy A, Day R (eds) Molecular imaging FRET microscopy and spectroscopy. Oxford University Press, New York, pp 126-145

Chen Y, Mauldin JP, Day RN, Periasamy A (2007) Characterization of spectral FRET imaging microscopy for monitoring nuclear protein interactions. J Microsc 228:139-152

Clegg RM (2002) FRET tells us about proximities, distances, orientations and dynamic properties. J Biotechnol 82:177-179

Dale R, Eisinger J, Blumberg WE (1979) The orientation freedom of molecular probes. The orientation factor in intermolecular energy transfer. Biophys J 26:161-193

Dobbie I, Linari M, Piazzesi G, Reconditi M, Koubassova N, Ferenczi MA, Lombardi V, Irving M (1998) Elastic bending and active tilting of myosin heads during muscle contraction. Nature 396(6709):383-387

Dominguez R, Freyzon Y, Trybus KM, Cohen C (1998) Crystal structure of a vertebrate smooth muscle myosin motor domain 
and its complex with the essential light chain: visualization of the pre-power stroke state. Cell 94(5):559-571

Ferenczi MA, Bershitsky SY, Koubassova N, Siththanandan V, Helsby WI, Panine P, Roessle M, Narayanan T, Tsaturyan AK (2005) The "roll and lock" mechanism of force generation in muscle. Struct 13:131-141

Garcia ID, Requejo-Isidro J, Webb MR, West TG, French P, Ferenczi MA (2010) Fluorescence lifetime imaging reveals that the ATP binding site of myosin in muscle senses force. Accepted for publication in Biophys $\mathbf{J}$

García ID, Lanigan P, Webb M, West TG, Requejo-Isidro J, Auksorius E, Dunsby C, Neil M, French P, Ferenczi MA (2007) Fluorescence lifetime imaging to detect actomyosin states in mammalian muscle sarcomeres. Biophys J 93:2091-2101

Geeves MA, Holmes KC (2005) The molecular mechanism of muscle contraction. Adv Protein Chem 71:161-193

Goldman YE, Simmons RM (1994) Control of sarcomere length in skinned muscle fibres of Rana temporaries during mechanical transients. J Physiol 350:497-518

Gordon GW, Berry G, Liang XH, Levine B, Herman B (1998) Quantitative fluorescence energy transfer measurements using fluorescence microscopy. Biophys J 74:2702-2713

Higuchi H, Yanagida T, Goldman YE (1995) Compliance of thin filaments in skinned fibres of rabbit skeletal muscle. Biophys $\mathbf{J}$ 69(3):1000-1010

Huxley HE (1969) The mechanism of muscular contraction. Science 164(886):1356-1365

Ishiwata S, Miki M, Shin I, Funatsu T, Yasuda K, dos Remedios CG (1997) Inter head distances in myosin attached to F-actin estimated by fluorescence energy transfer spectroscopy. Biophys J 73:895-904

Kast D, Espinoza-Fonseca LM, Yia C, Thomas DD (2010) Phosphorylation-induced structural changes in smooth muscle myosin regulatory light chain. Proc Natl Acad Sci 107:8207-8212

Kenworthy AK (2001) Imaging protein-protein interactions using fluorescence resonance energy transfer microscopy. Methods 24:289-296

Koubassova NA, Bershitsky SY, Ferenczi MA, Tsaturyan AK (2008) Direct modeling of X-ray diffraction pattern from contracting skeletal muscle. Biophys J 95:2880-2894

Koushik VS, Blank PS, Vogel SS (2009) Anomalous surplus energy transfer observed with multiple FRET acceptors. Ploss One 4(11):e8031

Liu J, Reedy MC, Goldman YE, Franzini-Armstrong C, Sasaki H, Tregear RT, Lucaveche C, Winkler H, Baumann BAJ, Squire JM, Irving TC, Reedy MK, Taylora KA (2004) Electron tomography of fast frozen, stretched rigor fibres reveals elastic distortions in the myosin cross bridges. J Struct Biol 147:268-282

Lorenz M, Popp D, Holmes KC (1993) Refinement of the F-actin model against $\mathrm{X}$-ray fibre diffraction data by the use of a directed mutation algorithm. J Mol Biol 234:826-836

McDowall AW, Hofmann W, Lepault J, Adrian M, Dubochet J (1984) Cryo-electron microscopy of vitrified insect flight muscle. J Mol Biol 178:105-111

Mendelson R, Morris EP (1997) The structure of the acto-myosin subfragment 1 complex: Results of searches using data from electron microscopy and X-ray crystallography. Proc Natl Acad Sci 94:8533-8538

Moss DJ, Trentham DR (1983) Distance measurement between the active site and cysteine 177 of the alkali one light chain of subfragment 1 from rabbit skeletal muscle. Biochem 22:5261-5270

Nashmi R, Fraser SE, Lester HA, Dickinson ME (2005) FRET measurements using multispectral imaging. In: Periasamy A,
Day R (eds) Molecular imaging FRET microscopy and spectroscopy. Oxford University Press, New York, pp 180-192

Neher RA, Neher E (2004) Applying spectral fingerprinting to the analysis of FRET images. Microsc Res Tech 64:185-195

Nyitrai M, Hild G, Bodis E, Lukacs A, Somogyi B (2000a) Flexibility of myosin-subfragment- 1 in its complex with actin as revealed by fluorescence resonance energy transfer. Eur $\mathrm{J}$ Biochem 267:4334-4338

Nyitrai M, Hild G, Lukacs A, Bodis E, Somogyi B (2000b) Conformational distributions and proximity relationships in the rigor complex of actin and myosin subfragment-1. J Biol Chem 275(4):2404-2409

Prochniewicz-Nakayama E, Yanagida T, Oosawa F (1983) Studies on conformation of F-actin in muscle fibres in the relaxed state, rigor, and during contraction using fluorescent phalloidin. J Cell Biol 97:1663-1667

Rayment I, Rypniewski WR, Schmidt-Bäse K, Smith R, Tomchick DR, Benning MM, Winkelmann DA, Wesenberg G, Holden HM (1993a) Three-dimensional structure of myosin subfragment-1: a molecular motor. Sci 261(5117):50-58

Rayment I, Holden HM, Whittaker M, Yohn CB, Lorenz M, Holmes KC, Milligan RA (1993b) Structure of the actin-myosin complex and its implications for muscle contraction. Sci 261(5117):58-65

Squire JH (1997) Architecture and function in the muscle sarcomere. Curr Opin Struct Biol 7:247-257

Stryer L (1978) Fluorescence energy transfer as a spectroscopic ruler. Ann Rev Biochem 47:819-846

Sun M, Rose MB, Ananthanarayanan S, Jacobs DJ, Yengo CM (2008) Characterization of the pre-force-generation state in the actomyosin cross-bridge cycle. Proc Natl Acad Sci 105(25): $8631-8636$

Tanner JW, Thomas DD, Goldman YE (1992) Transients in orientation of a fluorescent cross-bridge probe following photolysis of caged nucleotides in skeletal muscle fibre. J Mol Biol 223:185-203

Thaler C, Koushik S, Blank P, Vogel S (2005) Quantitative multiphoton spectral imaging and its use for measuring resonance energy transfer. Biophys J 89:2736-2749

Thirlwell H, Corrie JET, Trentham DR, Ferenczi MA (1994) Kinetics of relaxation from rigor of permeabilized fast-twitch skeletal fibres from the rabbit using a novel caged ATP and apyrase. Biophys J 67:2436-2447

Thomas DD (1987) Spectroscopic probes of muscle cross-bridge rotation. Ann Rev Physiol 49:691-709

Thomas DD, Cook R (1980) Orientation of spin-labelled myosin heads in glycerinated muscle fibres. Biophys J 32:891-906

Wallrabe H, Periasamy A (2005) Imaging protein molecules using FRET and FLIM microscopy. Curr Opin Biotechnol 16:19-27

Webb MR, Corrie JET (2001) Fluorescent coumarin-labelled nucleotides to measure ADP release from actomyosin. Biophys $\mathbf{J}$ 81:1562-1569

Webb MR, Reid GP, Munasinghe VRN, Corrie JET (2004) A series of related nucleotide analogues that aids optimization of fluorescence signals in probing the mechanism of P-loop ATPases, such as actomyosin. Biochem 43:14463-14471

Wouters FS, Verveer PJ, Bastiaens PIH (2001) Imaging biochemistry inside cells. Trends Cell Biol 11:203-211

Yang Y, Gourinath S, Kovacs M, Nyitray L, Reutzel R, Himmel DM, O’Neall-Hennessey E, Reshetnikova L, Szent-Gyorgyi AG, Brown JH, Cohen C (2007) Rigor-like structures from muscle myosin's reveal key mechanical elements in the transduction pathways of this allosteric motor. Struct 15:553-564

Zimmermann T (2005) Spectral imaging and linear unmixing in light microscopy. Adv Biochem Eng Biotechnol 95:245-265 